\title{
PECULIAR NEAR-NUCLEUS OUTGASSING OF COMET 17P/HOLMES DURING ITS 2007 OUTBURST
}

\author{
Chunhua Qi ${ }^{1}$, Michiel R. Hogerheijde ${ }^{2}$, David JewitT ${ }^{3}$, Mark A. Gurwell ${ }^{1}$, and David J. Wilner ${ }^{1}$ \\ ${ }^{1}$ Harvard-Smithsonian Center for Astrophysics, 60 Garden Street, MS 42, Cambridge, MA 02138, USA; cqi@ cfa.harvard.edu \\ ${ }^{2}$ Leiden Observatory, Leiden University, P.O. Box 9513, 2300 RA Leiden, The Netherlands \\ ${ }^{3}$ Department of Earth, Planetary and Space Sciences and Department of Physics and Astronomy, University of California at Los Angeles, \\ 595 Charles Young Drive East, Los Angeles, CA 90095, USA \\ Received 2014 September 16; accepted 2014 November 17; published 2015 January 20
}

\begin{abstract}
We present high angular resolution Submillimeter Array observations of the outbursting Jupiter family comet $17 \mathrm{P} /$ Holmes on 2007 October 26-29, achieving a spatial resolution of $2 . .5$, or $\sim 3000 \mathrm{~km}$ at the comet distance. The observations resulted in detections of the rotational lines CO 3-2, $\mathrm{HCN} 4-3, \mathrm{H}^{13} \mathrm{CN} 4-3, \mathrm{CS} 7-6, \mathrm{H}_{2} \mathrm{CO} 3_{1,2}-2_{1,1}$, $\mathrm{H}_{2} \mathrm{~S} 2_{2,0}-2_{1,1}$, and multiple $\mathrm{CH}_{3} \mathrm{OH}$ lines, along with the associated dust continuum at 221 and $349 \mathrm{GHz}$. The continuum has a spectral index of $2.7 \pm 0.3$, slightly steeper than blackbody emission from large dust particles. From the imaging data, we identify two components in the molecular emission. One component is characterized by a relatively broad line width $\left(\sim 1 \mathrm{~km} \mathrm{~s}^{-1}\right.$ FWHM) exhibiting a symmetric outgassing pattern with respect to the nucleus position. The second component has a narrower line width $\left(<0.5 \mathrm{~km} \mathrm{~s}^{-1}\right.$ FWHM) with the line center redshifted by $0.1-0.2 \mathrm{~km} \mathrm{~s}^{-1}$ (cometocentric frame), and shows a velocity shift across the nucleus position with the position angle gradually changing from $66^{\circ}$ to $30^{\circ}$ within the four days of observations. We determine distinctly different $\mathrm{CO} / \mathrm{HCN}$ ratios for each of the components. For the broad-line component we find $\mathrm{CO} / \mathrm{HCN}<7$, while in the narrow-line component, $\mathrm{CO} / \mathrm{HCN}=40 \pm 5$. We hypothesize that the narrow-line component originates from the ice grain halo found in near-nucleus photometry, believed to be created by sublimating recently released ice grains around the nucleus during the outburst. In this interpretation, the high $\mathrm{CO} / \mathrm{HCN}$ ratio of this component reflects the more pristine volatile composition of nucleus material released in the outburst.
\end{abstract}

Key words: comets: general - comets: individual (17P/Holmes) - radio lines: planetary systems submillimeter: planetary systems - techniques: interferometric

\section{INTRODUCTION}

Comets are thought to contain the most primitive material left over from the planetesimal building stage of the Sun's protoplanetary disk. Their physical and chemical composition provides an important link between nebular and interstellar processes (Ehrenfreund \& Charnley 2000; Mumma \& Charnley 2011). A fundamental goal of cometary research is to understand the origin and nature of cometary nuclei, in order to explore how they link back to the protoplanetary disk. The most abundant constituent of a cometary nucleus, water, is difficult to detect in ground-based observations due to interference from the Earth's atmospheric water vapor. However, many other molecules in cometary atmospheres can be detected, and these can be used to trace the distribution and kinematics of gas production from a comet. Of particular interest are parent molecules (those sequestered in the cometary nucleus), which sublime directly from the nucleus. Measurements of parent molecule production rates can reveal relative abundances in the nucleus itself and therefore provide information about the conditions under which the comet formed.

There are at least two distinct long-term reservoirs for cometary nuclei, the Oort Cloud and the Kuiper Belt. Measuring the composition from each of the reservoirs may shed light on their origins in the protoplanetary disk from which they formed. However, it is not straightforward to determine the composition of a comet without directly sampling the nucleus. Instead, we typically measure the coma emission of parent and daughter species released from the nucleus. This technique, while powerful, presents at least two problems. The first is that it is usually difficult to obtain spatial information on multiple species simultaneously, which is critical for the determination of abundance ratios due to the variable nature of comets (e.g., outflow asymmetries and jet activity). The second is that the chemical composition in the coma is not necessarily representative of the composition of the nucleus. For example, chemical reactions in the coma can affect the coma composition (e.g., HNC is likely to be formed in situ via chemistry in the inner coma; Mumma \& Charnley 2011). More seriously, thermal processing of the comet surface is expected to lead to preferential volatile loss, resulting in a gradient in composition with depth beneath the physical surface. Sublimation then proceeds from a range of depths, depending on the volatility of the ices, their permeability to gas flow, and the progression of thermally conducted heat moving into the interior. The gases escaping from the heated surface may not be representative of the bulk composition of the ices measured deep in the nucleus.

Radio interferometers possessing broad bandwidth coverage coupled with high spectral and spatial resolution can be used to examine spatial differences among parent molecules to probe the heterogeneity in the coma and nucleus (e.g., Blake et al. 1999). Simultaneous sampling of multiple parent molecules can test variations of abundances and abundance ratios associated with changes in the active area (Mumma \& Charnley 2011).

Jupiter family comets likely originated in the Kuiper Belt reservoir, although the precise source region within the Kuiper belt remains unclear (Volk \& Malhotra 2008). They have short orbital periods and average residence times in the inner solar system of $\sim 0.4 \mathrm{Myr}$ (Levison \& Duncan 1994). Volatile loss in previous orbits makes it especially difficult to determine their initial composition from observations of their comae; they can be very hard to observe because of their usually weak emission, and repeated passes near the Sun deplete volatiles from the surface and near-surface of the nucleus. However, a cometary outburst 
Table 1

Observational Parameters for Continuum Observations

\begin{tabular}{lcccccc}
\hline \hline $\begin{array}{l}\text { Date } \\
(\mathrm{UT})\end{array}$ & $\begin{array}{c}\lambda \\
(\mathrm{mm})\end{array}$ & $\begin{array}{c}\text { Frequency } \\
(\mathrm{GHz})\end{array}$ & Beam & P.A. & $\begin{array}{c}\text { Flux } \\
(\mathrm{mJy})\end{array}$ & $\begin{array}{c}\text { Offsets } \\
\left({ }^{\prime \prime}\right)\end{array}$ \\
\hline 2007 Oct 26.3-26.7 & 0.86 & 349 & $2^{\prime \prime} .7 \times 2^{\prime \prime} .1$ & 11.2 & $96.4 \pm 2.3$ & $(0.92,-0.26)$ \\
2007 Oct 27.3-27.7 & 1.35 & 221 & $3^{\prime \prime} .9 \times 3^{\prime \prime} .6$ & 40.8 & $30.3 \pm 0.6$ & $(0.59,-0.20)$ \\
2007 Oct 28.3-28.7 & 0.86 & 349 & $2^{\prime \prime} .4 \times 2^{\prime \prime} .1$ & 16.9 & $40.9 \pm 1.8$ & $(0.85,-0.26)$ \\
2007 Oct 29.3-29.7 & 0.86 & 348 & $2^{\prime \prime} .4 \times 2^{\prime \prime} .2$ & 13.7 & $48.8 \pm 1.4$ & $(0.83,-0.17)$ \\
\hline
\end{tabular}

potentially provides the opportunity to release fresh, minimally altered material from the interior of the nucleus. The unexpected outburst of Jupiter family comet 17P/Holmes in 2007 provided just such an opportunity to detect and study fresh material from the comet's deeper layers.

In this paper, we present Submillimeter Array (SMA) observations of the outburst of $17 \mathrm{P} /$ Holmes, in which the brightness increased from apparent magnitude $V \sim 17$ on UT 2007 October 23.3 (Hsieh et al. 2010) to $V \sim 2.0$ on UT 2007 October 25.0 ( $\mathrm{Li}$ et al. 2011), corresponding to the sudden ejection of ( 2 to 90$) \times 10^{10} \mathrm{~kg}$ of material (e.g., Ishiguro et al. 2010; $\mathrm{Li}$ et al. 2011). We use simultaneous imaging of HCN and CO from the outburst to identify two different components in the molecular emission. These components exhibit drastic differences in volatile composition as measured by $\mathrm{CO} / \mathrm{HCN}$, suggesting that one component samples pristine material from deep in the nucleus.

\section{OBSERVATIONS}

Observations of 17P/Holmes were made on four consecutive days between 2007 October 26 and 29 using the SMA eight antenna interferometer located atop Mauna Kea, Hawaii. The SMA operates in the 1.3 and $0.86 \mathrm{~mm}$ atmospheric transmission windows which cover rotational transitions from a variety of important cometary volatile species, such as $\mathrm{HCN}$, CO and CS. The SMA was used in the compact configuration, resulting in a spatial resolution of 2..5 at $349 \mathrm{GHz}$ and $4^{\prime \prime}$ at $221 \mathrm{GHz}$, corresponding to 2950 and $4730 \mathrm{~km}$ in linear scale at the Earth-comet distance of $1.63 \mathrm{AU}$. The primary beams are $31^{\prime \prime 2}$ and 49.'8 FWHM, respectively. The SMA operated in a double-sideband mode with an intermediate frequency band of 4-6 GHz which was sent over fiber optic transmission lines to 24 overlapping digital correlator bands (or "chunks") covering the $2 \mathrm{GHz}$ spectral window. Phase-switching techniques were used to separate and simultaneously recover both sidebands, resulting in $4 \mathrm{GHz}$ of total coverage ( $2 \mathrm{GHz}$ per sideband). Doppler-tracking of $17 \mathrm{P} /$ Holmes was performed so that the rapidly changing velocity of the comet, as defined by available ephemerides at the time of observations (specifically, JPL orbital solution \#K077/6, HORIZONS System), was compensated for in real time, so the velocity reported here is under the cometocentric velocity frame. At all frequencies, simultaneous dust continuum measurements were made using the line-free chunks. Tables 1 and 2 summarize the observational parameters for the continuum and spectral line setups used for each date of observation. In particular, observations on October 26, 28, and 29 focused on $\mathrm{HCN}$ and either $\mathrm{CO}$ or $\mathrm{CS}$ near $349 \mathrm{GHz}$, while those from October 27 aimed to detect $\mathrm{H}_{2} \mathrm{CO}, \mathrm{H}_{2} \mathrm{~S}, \mathrm{CH}_{3} \mathrm{OH}$ and the deuterated species HDO and DCN near $221 \mathrm{GHz}$.

Calibration of visibility phases and amplitudes was achieved with periodic snapshots of the quasar $0359+509$ at 20 minute intervals. Measurements of Uranus provided an absolute scale for calibration of the flux densities. The derived mean flux

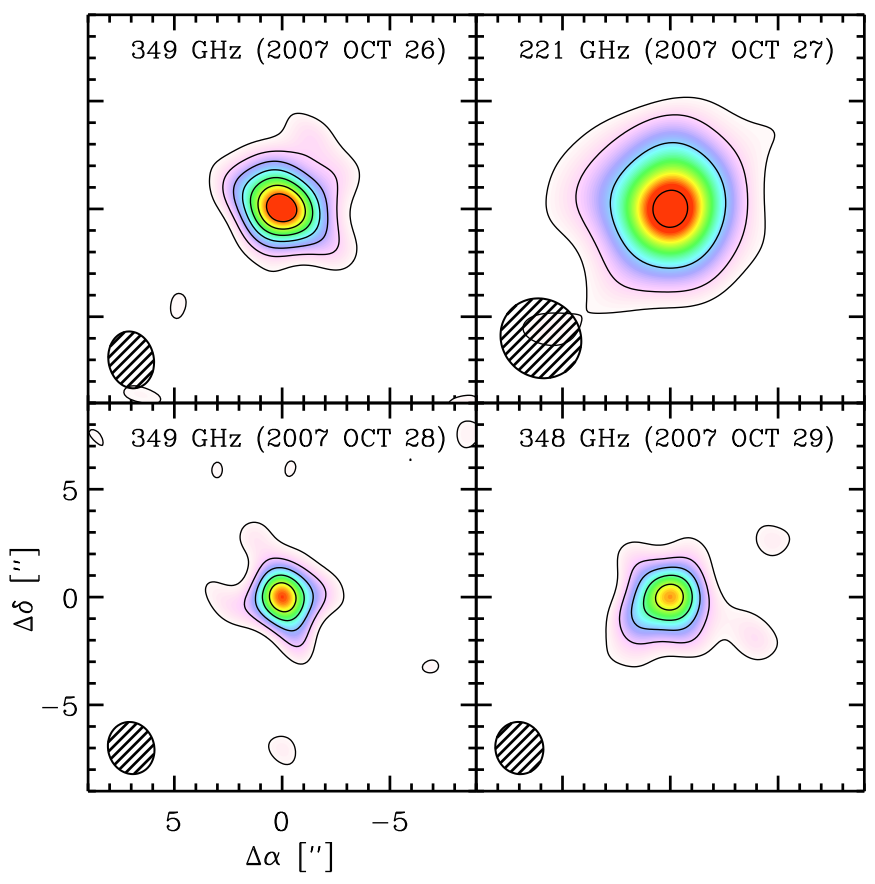

Figure 1. Interferometric maps of the (sub)mm continuum emission of comet $17 \mathrm{P} /$ Holmes obtained from 2007 October 26 to 29 UT with the SMA. The contours are $2.5 \mathrm{mJy}_{\text {beam }^{-1}}(1 \sigma) \times[2,4,6,8,10,12,14]$ (for October 26); $0.55 \mathrm{mJy} \mathrm{Beam}^{-1}(1 \sigma) \times[3,6,12,24]$ (for October 27); 2.0 $(1 \sigma) \times[2,4,6,8,10]$ (for October 28); $1.8(1 \sigma) \times[2,4,6,8,10]$ (for October 29).

densities of $0359+509$ at the time of the observations were $2.05 \mathrm{Jy}$ at $349 \mathrm{GHz}$ and $3.25 \mathrm{Jy}$ at $221 \mathrm{GHz}$. All data were phaseand amplitude-calibrated using the MIR software package. ${ }^{4}$ Continuum and spectral line maps were then generated and CLEANed using the MIRIAD software package.

\section{RESULTS}

Continuum emission at 221 and $349 \mathrm{GHz}$ is readily detected by the SMA. Figure 1 shows (sub)mm continuum emission maps of comet 17P/Holmes obtained from 2007 October 26 to 29 and integrated flux densities are reported in Table 1. The (sub)mm continuum emission probes the thermal radiation of $\mathrm{mm}$-sized dust particles in the coma and the peak of the emission is taken to reveal the location of the nucleus (e.g., Jewitt \& Luu 1992; Blake et al. 1999; Qi 2001).

Table 2 lists the observational parameters and resulting detections for the spectral line observations, while in Figure 2 the spectra from several molecular species are displayed for each day. These spectra are all extracted from a $10^{\prime \prime}$ square box centered on the nucleus, corresponding to the maximum area with significant emission. We note that the decrease of the

\footnotetext{
4 http://www.cfa.harvard.edu/ cqi/mircook.html
} 
Table 2

Observational Parameters for Line Observations

\begin{tabular}{|c|c|c|c|c|c|c|}
\hline Lines & $\begin{array}{c}\text { Rest Frequency } \\
(\mathrm{GHz})\end{array}$ & $\begin{array}{c}\text { Beam } \\
\left({ }^{\prime \prime}\right)\end{array}$ & $\begin{array}{c}\text { P.A. } \\
\left({ }^{\circ}\right)\end{array}$ & $\begin{array}{c}\text { Channel Spacing } \\
\left(\mathrm{km} \mathrm{s}^{-1}\right)\end{array}$ & $\begin{array}{l}\mathrm{FWHM}^{\mathrm{a}} \\
\left(\mathrm{km} \mathrm{s}^{-1}\right)\end{array}$ & $\begin{array}{l}\text { Int. Intensity } \\
\left(\mathrm{Jy} \mathrm{km} \mathrm{s}^{-1}\right)\end{array}$ \\
\hline \multicolumn{7}{|c|}{ UT: 2007 Oct 26.3-26.7 } \\
\hline $\mathrm{HCN} 4-3$ & 354.505 & $2.7 \times 2.1$ & 12.9 & 0.17 & 0.89 & $52.09[0.22]$ \\
\hline $\mathrm{H}^{13} \mathrm{CN} 4-3$ & 345.340 & $2.6 \times 2.0$ & 9.6 & 0.35 & 0.92 & $1.32[0.22]$ \\
\hline $\mathrm{CO} 3-2$ & 345.796 & $2.6 \times 2.0$ & 9.6 & 0.18 & 0.42 & $2.27[0.21]$ \\
\hline \multicolumn{7}{|c|}{ UT: 2007 Oct $27.3-27.7$} \\
\hline $\mathrm{H}_{2} \mathrm{CO} 3_{1,2}-2_{1,1}$ & 225.698 & $3.8 \times 3.2$ & 49.6 & 0.27 & 0.72 & $3.03[0.14]$ \\
\hline HDO $3{ }_{1,2}-22,1$ & 225.897 & $3.8 \times 3.2$ & 49.6 & 0.54 & $\ldots$ & {$[0.14]$} \\
\hline DCN 3-2 & 217.239 & $4.2 \times 3.9$ & -39.1 & 0.28 & $\ldots$ & {$[0.14]$} \\
\hline $\mathrm{H}_{2} \mathrm{~S} 22,0-2{ }_{1,1}$ & 216.710 & $4.2 \times 3.9$ & -39.1 & 0.56 & $\ldots$ & $1.36[0.14]$ \\
\hline $\mathrm{CH}_{3} \mathrm{OH} 5_{1,0}-4_{2,0}$ & 216.946 & $4.2 \times 3.9$ & -39.1 & 0.56 & $\cdots$ & $1.34[0.14]$ \\
\hline \multicolumn{7}{|c|}{ UT: 2007 Oct $28.3-28.7$} \\
\hline $\mathrm{HCN} 4-3$ & 354.505 & $2.5 \times 2.1$ & 19.4 & 0.086 & 0.69 & $48.79[0.16]$ \\
\hline $\mathrm{H}^{13} \mathrm{CN} 4-3$ & 345.340 & $2.4 \times 2.1$ & 14.2 & 0.35 & 0.68 & $1.07[0.16]$ \\
\hline $\mathrm{CO} 3-2$ & 345.796 & $2.4 \times 2.1$ & 14.2 & 0.088 & 0.50 & $1.75[0.15]$ \\
\hline $\mathrm{CH}_{3} \mathrm{OH} 13_{0,0}-12_{1,0}$ & 355.603 & $2.5 \times 2.1$ & 19.4 & 0.34 & $\cdots$ & $2.40[0.16]$ \\
\hline \multicolumn{7}{|c|}{ UT: 2007 Oct 29.3-29.7 } \\
\hline $\mathrm{HCN} 4-3$ & 354.505 & $2.4 \times 2.1$ & 15.6 & 0.17 & 0.65 & $50.16[0.21]$ \\
\hline CS 7-6 & 342.883 & $2.4 \times 2.2$ & 11.1 & 0.18 & 0.46 & $4.45[0.21]$ \\
\hline
\end{tabular}

Notes.

${ }^{\text {a }}$ Fitted with a Gaussian. Might not reflect the real line width.

b Integrated over $10^{\prime \prime}$ box.

continuum flux from October 26 to 29 is not smooth as the continuum flux on the 29th, which is slightly higher than that on the 28th. This is also accompanied by a small but significant increase in the velocity-integrated intensity level of HCN 4-3 as reported in Table 2 from the 28th to 29th. This is probably caused by the intermittent release of both big dust grains and volatiles sublimating from the small icy grains near the nucleus by the outburst.

For each day, we were able to cover several species within the $4 \mathrm{GHz}$ bandwidth (both upper and lower sidebands). This provides the opportunity to compare the extent of the emission from different species observed simultaneously in the coma during the outburst. On October 27, the SMA was tuned near $221 \mathrm{GHz}(1.4 \mathrm{~mm})$ in order to detect $\mathrm{H}_{2} \mathrm{CO}, \mathrm{H}_{2} \mathrm{~S}$, and $\mathrm{CH}_{3} \mathrm{OH}$, and search for the deuterated species HDO and DCN (see Table 2). Observations near $349 \mathrm{GHz}(0.86 \mathrm{~mm})$ of $\mathrm{HCN}$ with either CO or CS were obtained on October 26, 28, and 29. While several species at $1.4 \mathrm{~mm}$ were detected and the integrated intensities are reported in Table 2, the velocity resolution was insufficient for a detailed kinematic study, so in this paper we focus on the $\mathrm{HCN}$ and $\mathrm{CO} / \mathrm{CS}$ imaging and defer the analysis of $\mathrm{H}_{2} \mathrm{CO}, \mathrm{CH}_{3} \mathrm{OH}$, and $\mathrm{H}_{2} \mathrm{~S}$ to future work.

To better compare the emission of the different species, we regenerated channel images of the emission lines on a common velocity grid. Figures 3, 4, and 5 present the resampled $\mathrm{HCN}$ 4-3, CO 3-2, and CS 7-6 emission. The line width of HCN 4-3 is much broader than that of CO 3-2 or CS 7-6 for each day, and the emission of $\mathrm{HCN}$ is also more extended spatially than that of $\mathrm{CO}$ and $\mathrm{CS}$. Most of the HCN emission appears symmetric, consistent with expectations for isotropic outgassing. However, there are some deviations from this symmetric pattern. Some $\mathrm{HCN}$ emission (for example near $+0.4 \mathrm{~km} \mathrm{~s}^{-1}$ ) extend toward the northeast. In contrast, the CO 3-2 and CS 7-6 emission are significantly narrower in velocity width, and for both species the emission shifts position with velocity, showing a gradient across the continuum (nucleus) position. The peak emission from both lines is around the nucleus position, but about $0.1-0.2 \mathrm{~km} \mathrm{~s}^{-1}$ redshifted (cometocentric frame).

\section{ANALYSIS \\ 4.1. Continuum}

Continuum measurements at $0.86 \mathrm{~mm}$ (Table 1) from UT October 26,28 , and 29 show fading by a factor $\sim 2$, roughly corresponding to an exponential decay with e-folding time of $\sim 4$ days. By interpolation, the flux density at wavelength $\lambda_{0.86}=$ $0.86 \mathrm{~mm}$ on UT October 27 is estimated to be $S_{0.86}=68 \pm 7 \mathrm{mJy}$, which compares with the flux density at $\lambda_{1.35}=1.35 \mathrm{~mm}$ on this date of $S_{1.35}=30.3 \pm 0.6 \mathrm{mJy}$. The different beam sizes at $0.86 \mathrm{~mm}$ and $1.35 \mathrm{~mm}$ sample different volumes of the nearnucleus dust coma, requiring a correction. We assume that the dust density varies with the inverse square of the distance from the nucleus, in which case the quantity of dust intercepted by an aperture varies in proportion to $\phi$, the angular radius of the aperture. The effective aperture size is given by the projected beam which, for our data, is elliptical. We take the effective beam radius as $\phi=\left(\phi_{\max } \phi_{\min }\right)^{1 / 2}$, where $\phi_{\min }$ and $\phi_{\max }$ are the beam ellipse maximum and minimum radii from Table 1. We find $\phi_{0.86}=2^{\prime \prime} .3$ and $\phi_{1.35}=3$.'7. Then, the spectral index of the continuum of $17 \mathrm{P}$ in the wavelength range from 0.86 to $1.35 \mathrm{~mm}$ is given by

$$
\alpha=\frac{\log \left[\left(S_{1.35} / S_{0.86}\right)\left(\phi_{0.86} / \phi_{1.35}\right)\right]}{\log \left(\lambda_{1.35} / \lambda_{0.86}\right)} .
$$

By substitution, we obtain $\alpha=2.7 \pm 0.3$, where the quoted uncertainty mainly reflects the error in $S_{0.86}$ on UT October 27 introduced by the interpolation from adjacent dates. Evidently, $\alpha$ is slightly larger than the expected blackbody spectral index in the Rayleigh-Jeans regime, namely $\alpha=2$. However, 

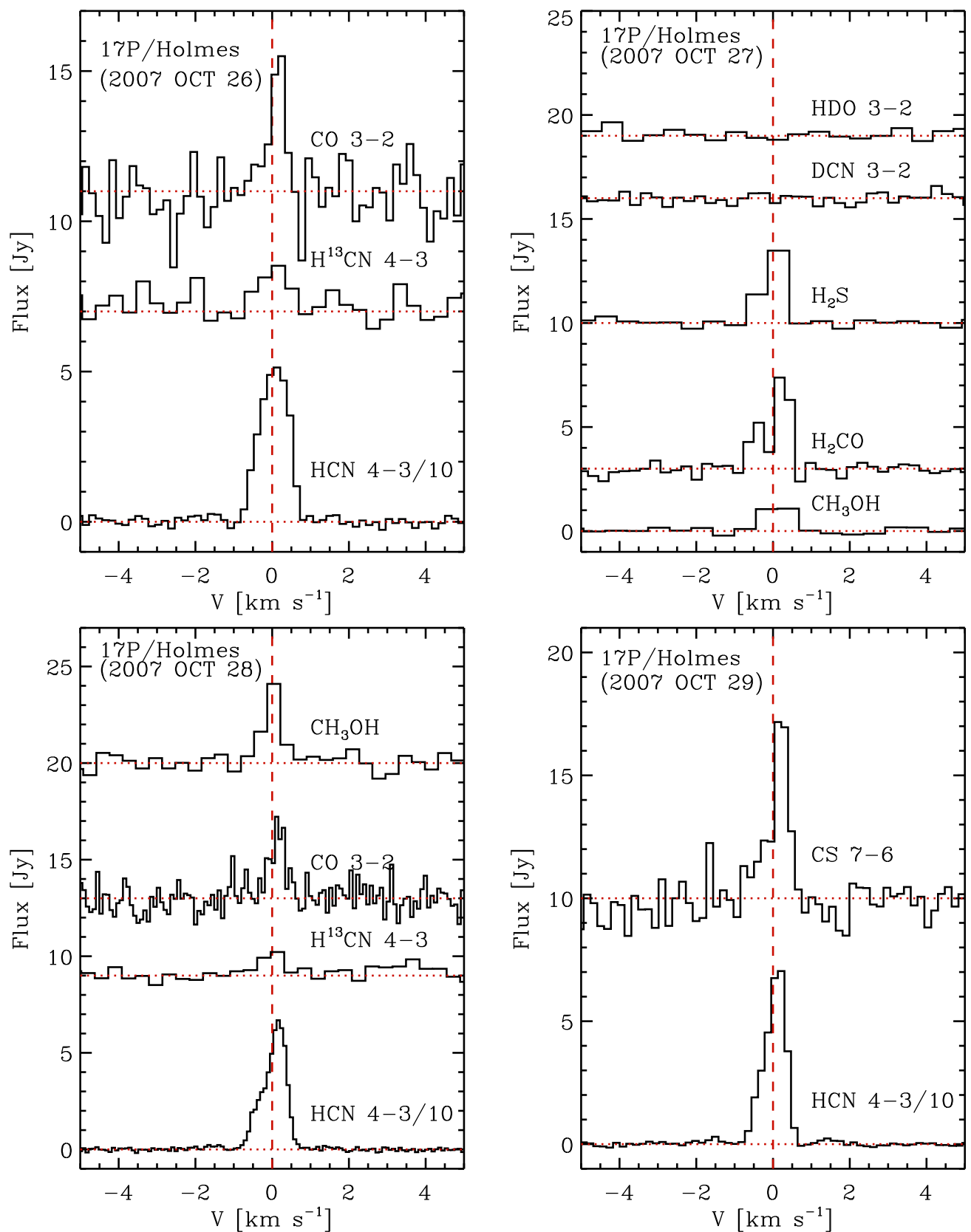

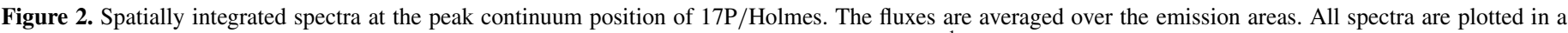
cometocentric velocity frame. The vertical dashed line marks the cometocentric velocity of $0 \mathrm{~km} \mathrm{~s}^{-1}$.

it is consistent with earlier measurements of dust in comet $\mathrm{C} /$ Hale-Bopp, which gave $\alpha=2.60 \pm 0.13$ (Jewitt \& Matthews 1999). Values of $\alpha$ near 2 indicate that the continuum cross-section is dominated by optically large dust particles $(2 \pi a / \lambda>1$, corresponding to particle radii $a \gtrsim$ $200 \mu \mathrm{m})$.

We used Equation (6) of Jewitt \& Matthews (1999) to estimate the mass loss rate from $S_{1.35}$ (Table 1), obtaining $d M / d t=2.6 \times 10^{6} \mathrm{~kg} \mathrm{~s}^{-1}$ on UT October 27 . This value, about 1000 times larger than typical of Jupiter family comets at the same heliocentric distance, may be compared with the peak mass loss rate three days earlier on UT 2007 October 24.5, estimated from optical continuum data as being up to $1.4 \times 10^{7} \mathrm{~kg} \mathrm{~s}^{-1}$ (Li et al. 2011). On the other hand, the (gas) production rate five days later, on UT 2007 November 1, had fallen to $2 \times$ $10^{4} \mathrm{~kg} \mathrm{~s}^{-1}$ (Schleicher 2009). Even accounting for the decay of the brightness of 17P between October 27 and November 1, it appears that $17 \mathrm{P}$ was an extremely dust-rich comet.

\subsection{Symmetric Outgassing Model}

Figures 3, 4, and 5 suggest that much of the HCN emission toward 17P/Holmes originated from symmetric outgassing. This emission typically has a relatively large linewidth (FWHM $\sim$ $\left.1 \mathrm{~km} \mathrm{~s}^{-1}\right)$. There is also clear evidence that some $\mathrm{HCN}$ emission does not follow this symmetric pattern. The CO 3-2 and CS 7-6 emission are dominated by emission asymmetric with respect to the systemic velocity of the comet $\left(0 \mathrm{~km} \mathrm{~s}^{-1}\right.$ in the cometocentric frame), and shows smaller line widths of FWHM $<$ $0.5 \mathrm{~km} \mathrm{~s}^{-1}$. Is there a relation between the asymmetries in the $\mathrm{HCN}$ line and the narrow $\mathrm{CO}$ and $\mathrm{CS}$ emission? To answer this question requires detailed modeling of the $\mathrm{HCN}$ emission. 


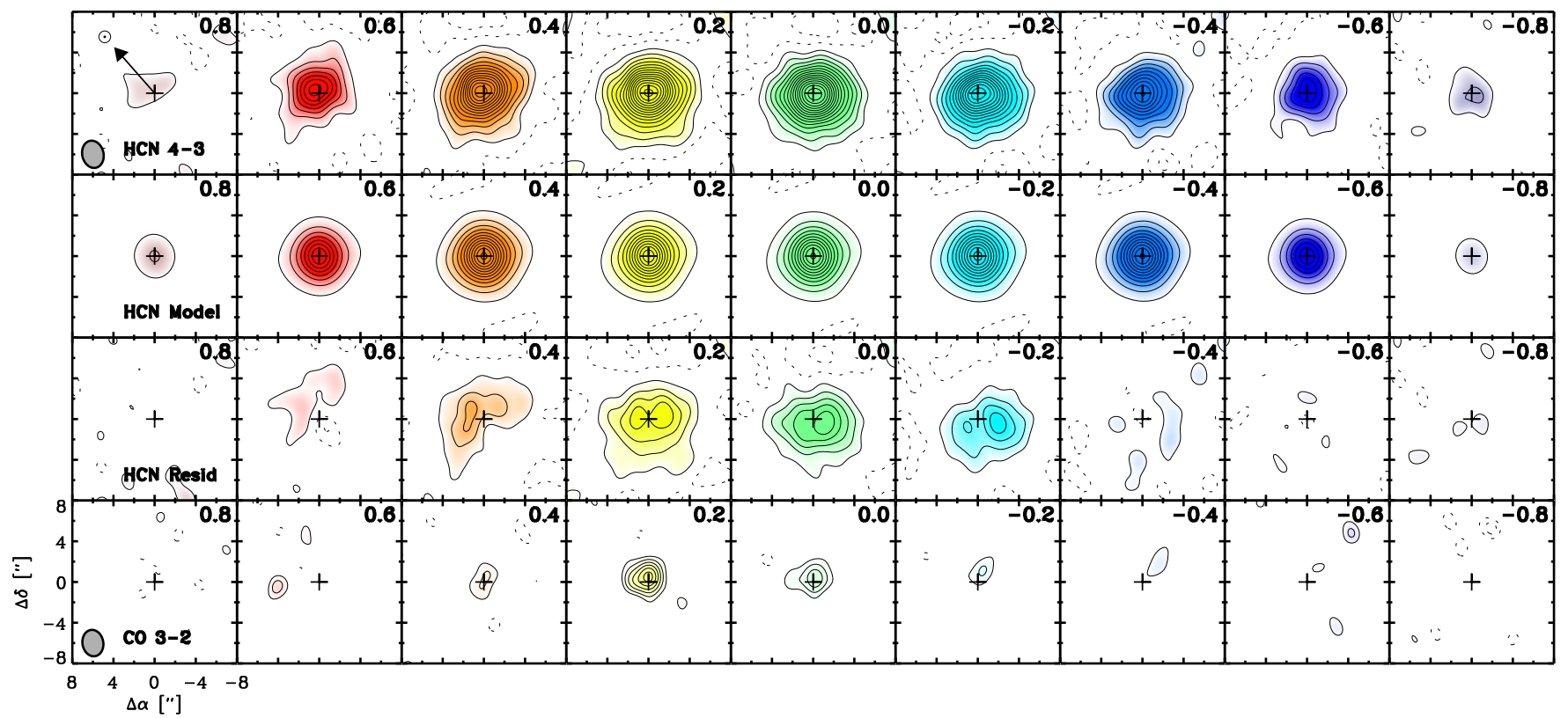

Figure 3. SMA observations of molecular emission from Comet 17P/Holmes on 2007 October 26, two days after the onset of outburst. The residual of the HCN 4-3 emission (third row), obtained by subtracting the best-fit symmetric outgassing model (second row) from the data (first row), is likely to created by sublimating recently released ice grains around the nucleus during the outburst. The extent of the $\mathrm{CO} 3-2$ emission (fourth row) is consistent with the residual of $\mathrm{HCN}$ emission, indicating a higher volatile content in this component. For HCN emission, the contours start with $2 \sigma$ and each following contour step is $3 \sigma\left(1 \sigma=0.3 \mathrm{Jy}\right.$ beam ${ }^{-1}$ ). For $\mathrm{CO}$ emission, the contours start with $2 \sigma$ and step in $1 \sigma$. The cross marks the position of the nucleus and arrows show the direction of the Sun. North is the positive $Y$-axis and east is the positive $X$ axis.

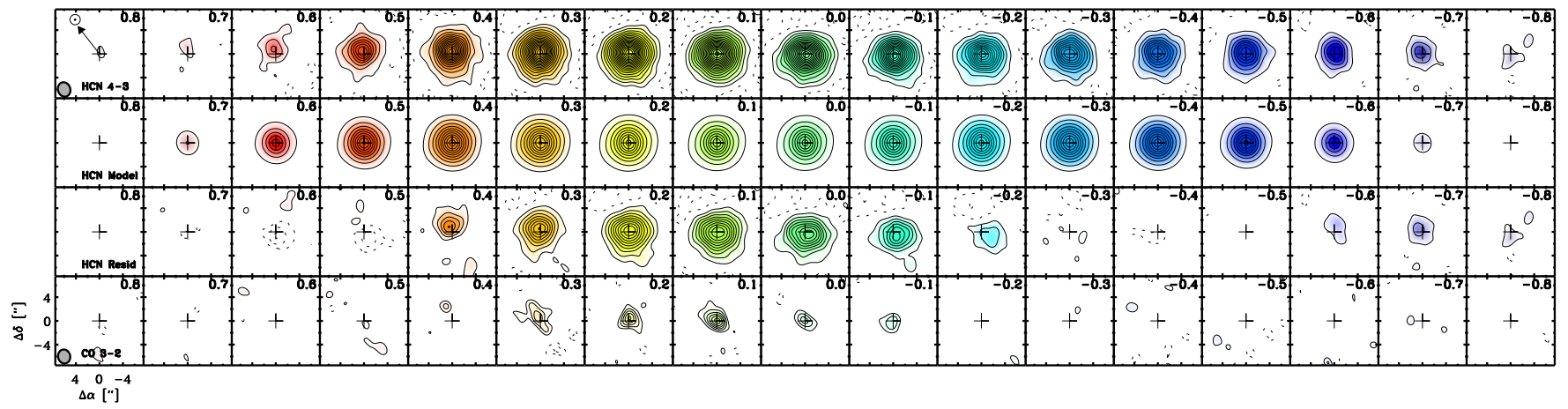

Figure 4. Same as Figure 3, but for 2007 October 28.

The modeling analysis consists of the following steps. First, we construct a symmetric outgassing model for HCN. Second, we subtract the symmetric outgassing model from the data, and compare the subtracted $\mathrm{HCN}$ residual with the CO 3-2 and CS 7-6 emission to determine if they share the same origin. Finally, we investigate any compositional differences between the symmetric and asymmetric parts of the coma.

For a first-order analysis of the distribution of $\mathrm{HCN}$ in the coma, we assume that the density in the coma drops with distance from the nucleus, which is the only source for HCN. The distribution of density as a function of radius can be approximated using the Haser model with a constant expansion velocity $v_{\text {exp }}$. The modeling procedure is similar to that presented in Hogerheijde et al. (2009), adapted to the geometrical circumstances of $17 \mathrm{P} /$ Holmes. We calculate line formation and molecular excitation in the coma using an adapted version of the Accelerated Monte Carlo code by Hogerheijde \& van der Tak (2000), in which we consider excitation via collisions with water and electrons, spontaneous and induced emission of line photons, and absorption of line and continuum photons, including those of the solar infrared spectrum (see Section 4.2 of Hogerheijde et al. 2009 for details of molecular excitation calculation). For the main collisional partner, we adopt a water production rate $Q\left(\mathrm{H}_{2} \mathrm{O}\right)=7,4$, and $3 \times 10^{29} \mathrm{~mol} \mathrm{~s}^{-1}$, appropriate for October 26, 28, and 29 (Dello Russo et al. 2008). For the gas temperature, we adopt a constant kinetic temperature of $45 \mathrm{~K}$, based on the results of BockeléeMorvan et al. (2008).

$\mathrm{HCN}$ has a hyperfine structure due to the nuclear quadruple moment of ${ }^{14} \mathrm{~N}$. We adopt the six HCN hyperfine components of the 4-3 transition and their relative intensities from the JPL Molecular Spectroscopy database listed in the Table 3. Relative populations between the hyperfine levels are assumed to be in LTE. The HCN emission is not very optically thick. For example on October 28, the integrated intensity of the central four hyperfine components is $46.30 \mathrm{Jy} \mathrm{km} \mathrm{s}{ }^{-1}$, and the outer hyperfine component $44_{3}-3_{3}$ (well separated from the others) is $1.25 \mathrm{Jy} \mathrm{km} \mathrm{s}^{-1}$. So the measured ratio of this component over the central four combined is $0.026 \pm 0.003$, close to the expected ratio of 0.022 for optically thin emission. After construction of the emission model, we multiply it by the appropriate primary beams and resample with the $u v$ data using the actual SMA 


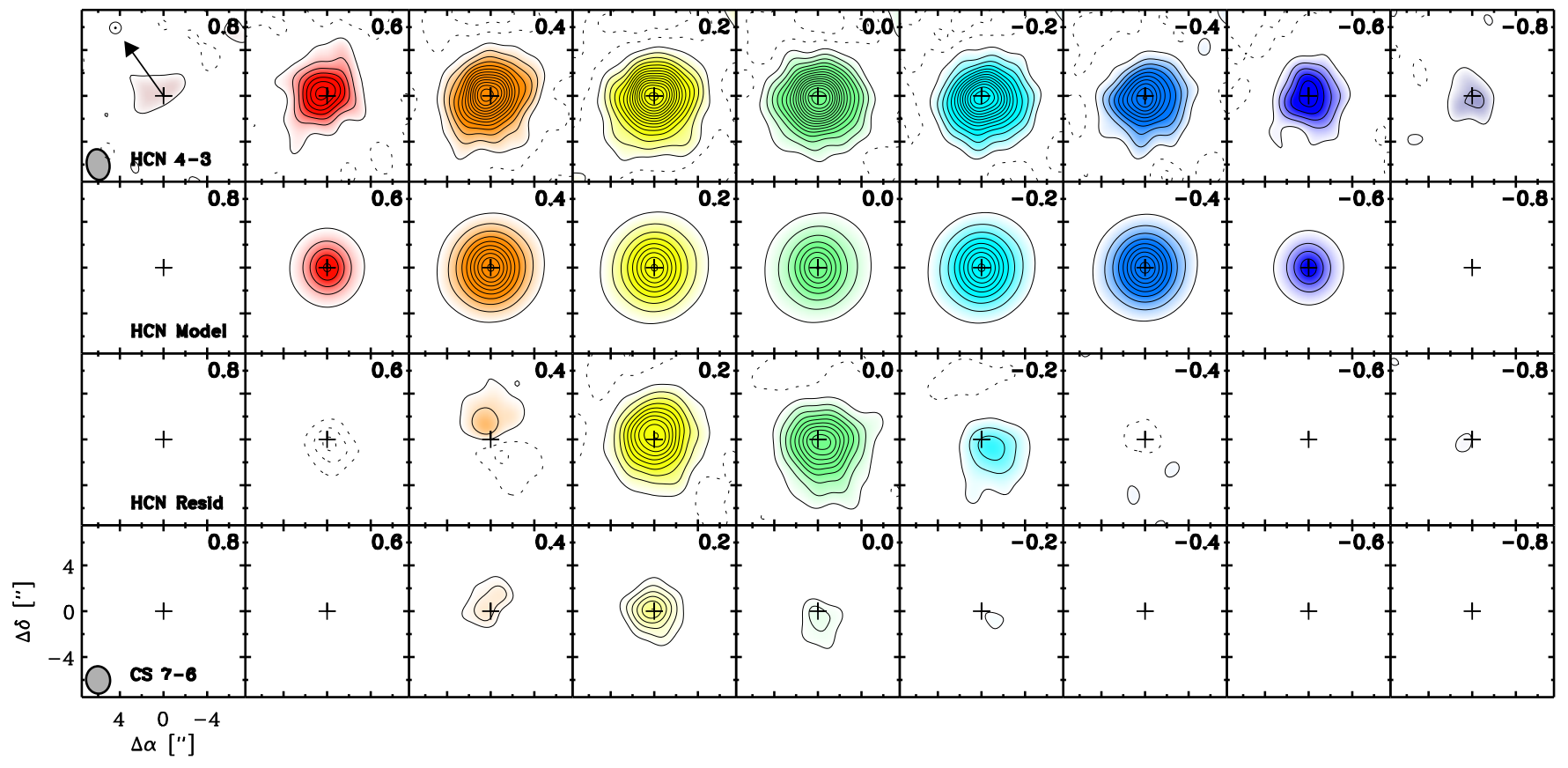

Figure 5. Same as Figure 3, but for 2007 October 29. For CS emission, the contours start with $2 \sigma$ and step in $1 \sigma\left(1 \sigma=0.3 \mathrm{Jy} \mathrm{beam}^{-1}\right)$.

Table 3

Spectroscopic Values for HCN 4-3 Hyperfine Components

\begin{tabular}{lccc}
\hline \hline J F & $\mathrm{J}^{\prime} \mathrm{F}^{\prime}$ & $\begin{array}{c}\text { Frequency } \\
(\mathrm{GHz})\end{array}$ & Rel. Intensity \\
\hline 44 & 34 & 354.5038689 & 0.021 \\
43 & 32 & 354.5053670 & 0.24 \\
44 & 33 & $354.5054778^{\mathrm{a}}$ & 0.31 \\
45 & 34 & 354.5055234 & 0.41 \\
43 & 34 & 354.5058468 & $5 \times 10^{-4}$ \\
43 & 33 & 354.5074558 & 0.021 \\
\hline
\end{tabular}

Note. ${ }^{\text {a }}$ Reference frequency.

antenna positions to generate model visibilities. To find the best-fitting description of the symmetric emission, we only consider emission outside the cometocentric velocity range of -0.2 to $+0.4 \mathrm{~km} \mathrm{~s}^{-1}$, corresponding to the range outside where the narrow $\mathrm{CO}$ and $\mathrm{CS}$ emission dominate. Using the comet model and the excitation calculation described above, we compute a grid of synthetic $\mathrm{HCN}$ visibility data sets over a range of $Q_{\mathrm{HCN}}$ and $v_{\exp }$ values and compare with the observations. The best-fit model is obtained by minimizing the $\chi^{2}$, the weighted difference between the data and the model with the real and imaginary part of the complex visibility measured in the $(u, v)$-plane sampled by the HCN observations. Our analysis is performed in the visibility domain rather than the image domain, to avoid the nonlinear effects of the imaging and deconvolution process.

The best-fit $\mathrm{HCN}$ production rates and expansion velocities are derived and listed in Table 4. Figures 3, 4, and 5 present comparisons between the observed channel maps and the bestfit model. The model reproduces the main features of symmetric pattern of the HCN emission. The line optical depth at center is around 0.5-0.6, consistent with the optical depth assessed above using the hyperfine components ratio. Figure 6 shows the $\chi^{2}$ surfaces for the $Q_{\mathrm{HCN}}$ versus $V_{\exp }$ values, which enables us to quantify the uncertainties associated with the model production rates and the expansion velocities fit for the three dates. The bestfit $\mathrm{HCN}$ production rates decrease from the 26th to the 29th and
Table 4

HCN Symmetric Outgassing Model Fitting Result ${ }^{\mathrm{a}}$

\begin{tabular}{lcc}
\hline \hline Date & $v_{\exp }\left(\mathrm{km} \mathrm{s}^{-1}\right)$ & $Q_{\mathrm{HCN}}\left(10^{26} \mathrm{~mol} \mathrm{~s}^{-1}\right)$ \\
\hline 2007 Oct 26 & $0.46[0.01]$ & $12.5[1.0]$ \\
2007 Oct 28 & $0.44[0.01]$ & $8.0[0.3]$ \\
2007 Oct 29 & $0.38[0.01]$ & $6.3[0.2]$ \\
\hline
\end{tabular}

Note. ${ }^{a}$ Symmetric outgassing model fitting on velocity range outside of -0.2 to $+0.4 \mathrm{~km}^{-1}$, i.e., not including the asymmetric narrow-line component of the $\mathrm{HCN}$ emission.

the outflow expansion velocity drops quickly. However, there are significant residuals between the data and model. Figure 7 shows the spectra of the symmetric HCN line and the residual, compared with the full spectra of HCN line taken on October 28, demonstrating the narrow line width of the residual compared to the symmetric model.

Using this symmetric model, we need $Q_{\mathrm{HCN}}=(1.20 \pm 0.01) \times$ $10^{27}$ and $Q_{\mathrm{H}^{13} \mathrm{CN}}=(1.5 \pm 0.3) \times 10^{25} \mathrm{~s}^{-1}$ to match the October $28 \mathrm{HCN}$ and $\mathrm{H}^{13} \mathrm{CN} 4-3$ integrated intensities (see Table 2). Therefore we derive $\mathrm{H}^{12} \mathrm{CN} / \mathrm{H}^{13} \mathrm{CN}=80 \pm 16$, which is consistent with the nominal terrestrial value of 89 and with the measurement $\left(\mathrm{H}^{12} \mathrm{CN} / \mathrm{H}^{13} \mathrm{CN}=114 \pm 26\right)$ by BockeléeMorvan et al. (2008). We note that the production rates reported in Table 4 are derived to fit only the part of the HCN lines contributed by the symmetric outgassing. It is not possible to fit the full $\mathrm{HCN}$ and $\mathrm{H}^{13} \mathrm{CN}$ emission using only the symmetric model. However, as long as we can generate similar integrated intensities and treat both lines in the same way, this should give reasonable estimates of the abundance ratio. With the same method, we obtain $Q_{\mathrm{HCN}}=1.5 \times 10^{27}$ for 2007 October 27 by interpolating the HCN production rates between October 26 and October 28, and the upper limit of $Q_{\mathrm{DCN}}=1.2 \times 10^{25}$ to achieve $0.42 \mathrm{Jy} \mathrm{km} \mathrm{s}^{-1}$ flux density ( $3 \sigma$ upper limit) of DCN 3-2 emission obtained on 2007 October 27, and derive $\mathrm{DCN} / \mathrm{HCN}<8 \times 10^{-3}$, consistent with the bulk $\mathrm{D} / \mathrm{H}$ ratio in HCN measured from comet Hale-Bopp $\left(2.3 \pm 0.4 \times 10^{-3}\right.$; 

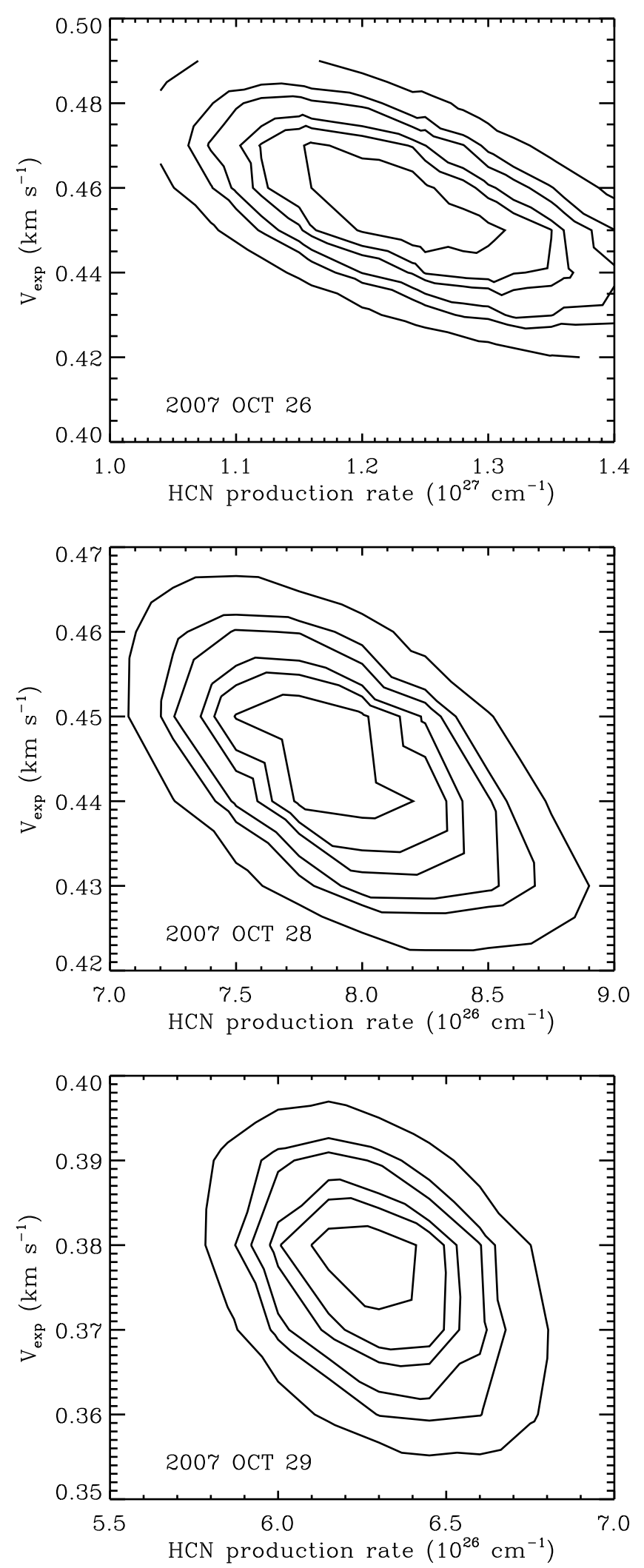

Figure 6. Iso- $\chi^{2}$ surfaces of $Q_{\mathrm{HCN}}$ vs. $v_{\mathrm{exp}}$. Contours correspond to the $1 \sigma-6 \sigma$ errors.

Meier et al. 1998). However, we cannot yet separate the contribution from the freshly released material in the nucleus as in Blake et al. (1999) due to the limited sensitivity.

The derived bulk HCN production rate of $1.2 \times 10^{27} \mathrm{~s}^{-1}$ on October 28 and an interpolated value of $1.5 \times 10^{27} \mathrm{~s}^{-1}$ on

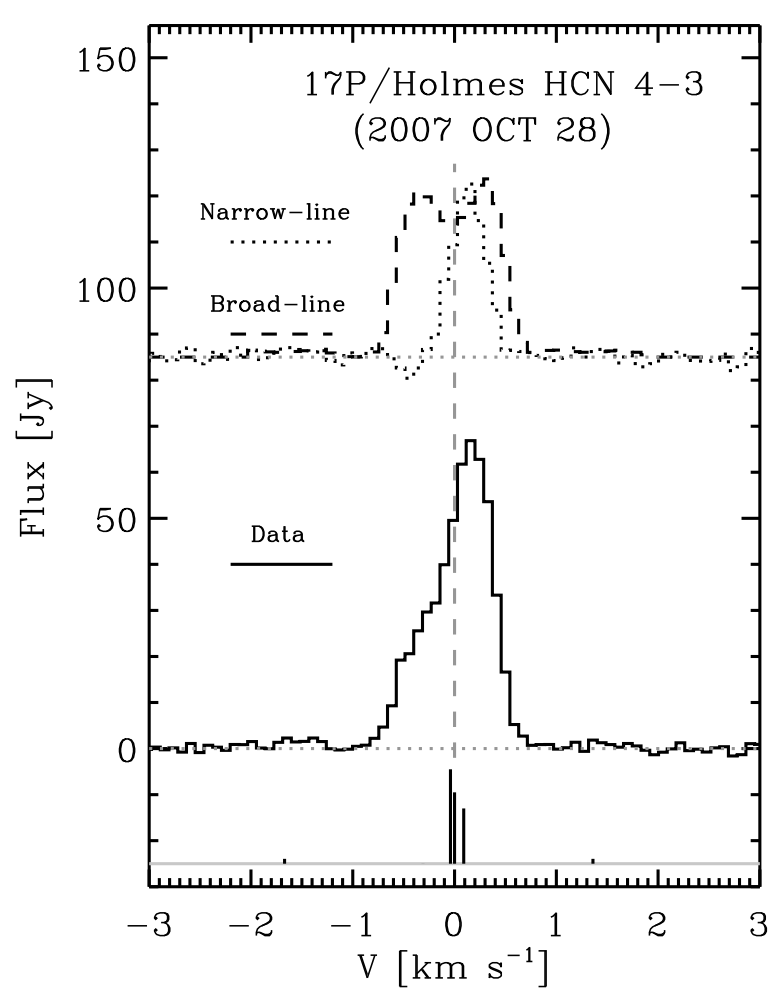

Figure 7. The broad-line component (dashed line) and narrow-line component (dotted line), compared with the full $\mathrm{HCN}$ data spectra (solid line) from 17P/Holmes on 2007 October 28 UT. The positions and relative intensities of the hyperfine components of HCN 4-3 transition are shown at the bottom. The broad-line component is retrieved from the best-fit symmetric outgassing model with $v_{\mathrm{exp}}=0.44 \mathrm{~km} \mathrm{~s}^{-1}$ and $Q_{\mathrm{HCN}}=8 \times 10^{26} \mathrm{~s}^{-1}$. The narrow-line component is derived from the residual of the $\mathrm{HCN}$ emission subtracted from the best-fit symmetric outgassing model. The vertical dashed line marks the cometocentric velocity of $0 \mathrm{~km} \mathrm{~s}^{-1}$.

October 27 are generally consistent with the values derived from single dish observations $\left(2.0 \times 10^{27}\right.$, IRAM $30 \mathrm{~m}$; BockeléeMorvan et al. 2008) and infrared observations $\left(2.4 \times 10^{27}\right.$, NIRSPEC; Dello Russo et al. 2008) derived on October 27, considering the uncertainties involved in the measurements and the use of different aperture sizes.

\subsection{Narrow-line Component}

As shown in Figure 7, the linewidth of the residual HCN emission is much smaller than those in the symmetric outgassing models. The spatio-kinematic characteristics of the residual $\mathrm{HCN}$ emission are very similar to those of the $\mathrm{CO} 3-2$ and CS 7-6 observed simultaneously on those days. We will refer to this component as the narrow-line component. The main characteristics that separate it from the broad-line emission are:

1. The narrow-line component occupies a velocity range from -0.2 to $+0.4 \mathrm{~km} \mathrm{~s}^{-1}$, much narrower than the broad-line component associated with symmetric outgassing that emits from -0.8 to $+0.8 \mathrm{~km} \mathrm{~s}^{-1}$.

2. The line center (where the image peak coincides with the peak of the mm continuum emission or nucleus position) for the HCN 4-3 residuals, CO 3-2, and CS 7-6 are all around $0.1-0.2 \mathrm{~km} \mathrm{~s}^{-1}$ redshifted, while the line center velocity of the broad-line component is $0 \mathrm{~km} \mathrm{~s}^{-1}$.

3. The narrow-line component shows emission that is redshifted toward the northeast and blueshifted toward the southwest relative to the $\mathrm{mm}$ continuum peak. Figure 8 shows the redshifted (integrated from +0.3 to 


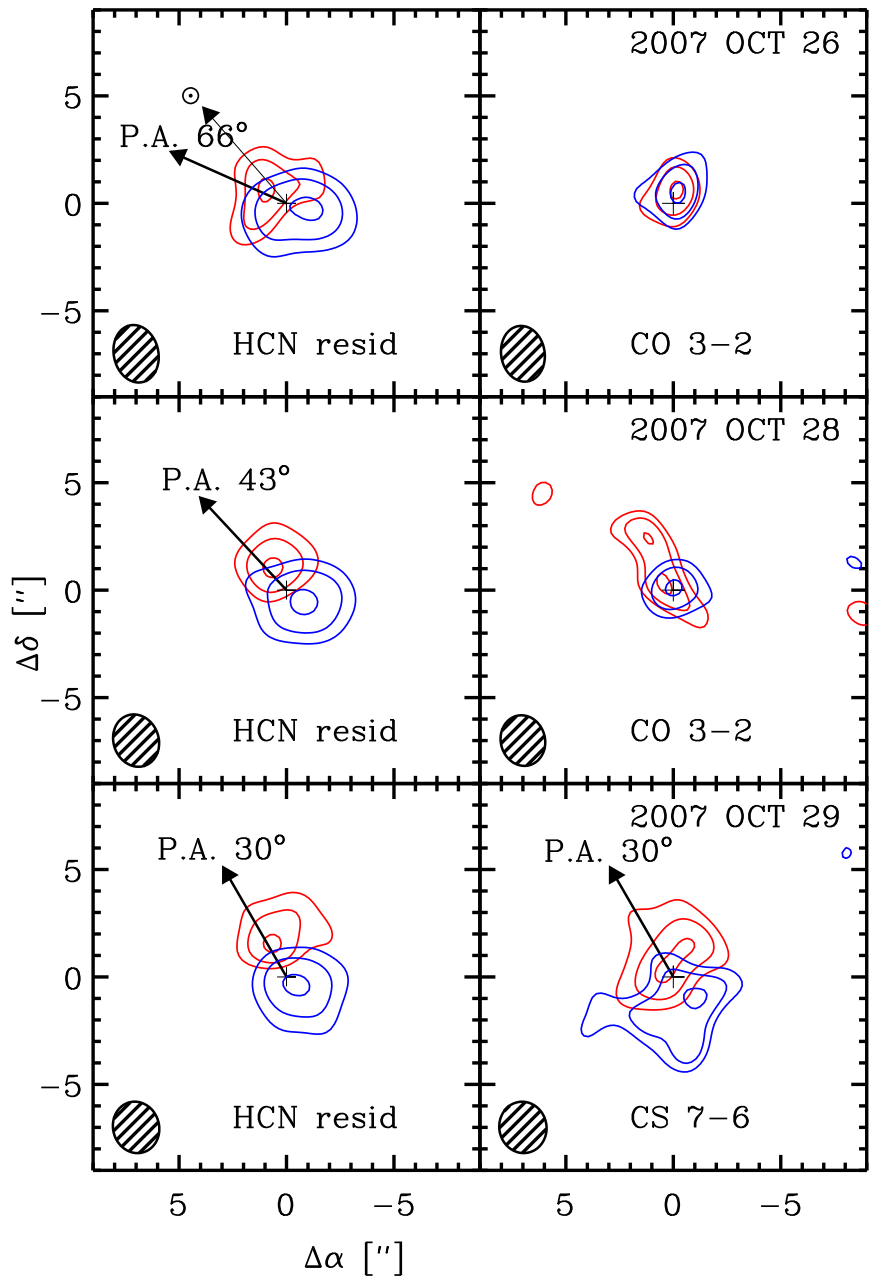

Figure 8. Integrated intensity images redshifted (integrated from 0.3 to $0.6 \mathrm{~km} \mathrm{~s}^{-1}$ ) vs. blueshifted (integrated from -0.3 to $0 \mathrm{~km} \mathrm{~s}^{-1}$ ) for $\mathrm{HCN}$ 4-3 residuals, CO 3-2, and CS 7-6 on 2007 October 26-29. The contours are at $50 \%, 70 \%$, and $95 \%$ of the peak value. The cross marks the peak of the continuum emission, which locates the position of the nucleus. P.A. is measured from the peak of the blueshifted image to that of the redshifted image. The Sun direction is shown for 2007 October 26 and the solar direction only changed 4.1 clockwise from October 26 to 29 .

$+0.6 \mathrm{~km} \mathrm{~s}^{-1}$ ) and the blueshifted (integrated from -0.3 to $0 \mathrm{~km} \mathrm{~s}^{-1}$ ) emission of the $\mathrm{HCN} \mathrm{4-3} \mathrm{residuals,} \mathrm{compared}$ with the CO 3-2 and CS 7-6 emission on 2007 October 26-29. Especially on the 29th, the position angle (P.A.) of the $\mathrm{HCN}$ residual is the same as that of CS 7-6. The velocity shift of CO 3-2 is not as obvious on 26th but it does show a similar extension as the HCN residual on 28th.

The volatiles in the narrow-line component likely share the same origin in the outbursting coma of $17 \mathrm{P} /$ Holmes. The P.A. of the red/blue gradient of the narrow emission changed gradually from $66^{\circ}$ to $30^{\circ}$ during the period of observations, although in the same sense, this is a much larger shift than that of the P.A. of the line toward the Sun, which only changed from 41.0 to 36.9 . We propose that the (residual) $\mathrm{HCN}, \mathrm{CO}$, and CS emission of the narrow component may come from volatiles released from very small grains of pure ice freshly released from the nucleus. Without absorptive dust, the ice warms up at a much slower rate. $\mathrm{CO}$ might be released faster than HCN and CS (presumably a product of $\mathrm{CS}_{2}$ in the ice), accounting for the morphological difference between $\mathrm{CO}$ and $\mathrm{CS}$ (and the $\mathrm{HCN}$ residuals). We hypothesize that the narrow-line component originates from the ice grain halo found in the near-nucleus photometry which is believed to be created by sublimating ice grains around the nucleus due to the outburst (Stevenson \& Jewitt 2012). Based on time-series photometry of $17 \mathrm{P} /$ Holmes before the outburst, Snodgrass et al. (2006) estimated a rotation period for 17P/ Holmes of $7.2<P_{\text {rot }}<12.8 \mathrm{hr}$. If the change of the P.A. of the narrow-line component was caused by the rotation of the nucleus, the gradual change from $66^{\circ}$ to $30^{\circ}$ from 26 th to 29 th implies a nucleus rotational period of $11.8 \mathrm{hr}$ (clockwise in the plane perpendicular to the line of the sight) or $12.2 \mathrm{hr}$ (anticlockwise). We discuss the nature of this narrow-line component further in the discussion section.

\section{4. $\mathrm{CO} / \mathrm{HCN}$ Ratios}

Here we investigate the $\mathrm{CO} / \mathrm{HCN}$ ratios within the two components based on the data obtained on 2007 October 28, which had the finest spectral resolution for both HCN 4-3 and CO 3-2. For HCN, the parameters of the broad-line component come from the best-fit symmetric outgassing model as described above, by fitting the data avoiding the velocity range from -0.2 to $+0.4 \mathrm{~km} \mathrm{~s}^{-1}$. The best-fit production rate is $8.0 \times 10^{26} \mathrm{~s}^{-1}$ and the expansion velocity $0.44 \mathrm{~km} \mathrm{~s}^{-1}$. The narrow-line component is retrieved from the residual $\mathrm{HCN}$ emission after subtraction of the best-fit symmetric outgassing model. Figure 4 and 7 show the simulated HCN images and spectra of the two components compared with the data. As shown in the figures the narrowline component mainly emits over the velocity range of -0.2 to $+0.4 \mathrm{~km} \mathrm{~s}^{-1}$, which is consistent with the $\mathrm{CO}$ emission obtained simultaneously. This indicates that most of the $\mathrm{CO}$ emission, if not all, must originate from the narrow-line component.

In order to estimate how much the broad-line component contributes to the $\mathrm{CO}$ emission, we make use of the emission avoiding the velocity range from -0.2 to $+0.4 \mathrm{~km} \mathrm{~s}^{-1}$, similar to the HCN analysis. However, since the signal is too weak for the $\mathrm{CO}$ emission, we integrate the emission with velocity between -0.8 to $+0.8 \mathrm{~km} \mathrm{~s}^{-1}$, again avoiding the velocity range from -0.2 to $+0.4 \mathrm{~km} \mathrm{~s}^{-1}$, which is clearly dominated by the narrowline component. Figure 9 shows the integrated intensity image, which does not show any significant emission left with the noise level at $0.09 \mathrm{Jy} \mathrm{beam}^{-1} \mathrm{~km} \mathrm{~s}^{-1}$. The upper limit of the broadline component contribution can be obtained by simulating $\mathrm{CO}$ emission with the same broad-line expansion velocity as determined in $\mathrm{HCN}$ emission, i.e., $0.44 \mathrm{~km} \mathrm{~s}^{-1}$, and matching the $1 \sigma$ integrated flux level within the same velocity range. This is achieved with $Q_{\mathrm{CO}}=5.0 \times 10^{27} \mathrm{~s}^{-1}$. Figure 9 compares the integrated intensity of the simulated image with that of $\mathrm{CO}$ data and Figure 10 demonstrates the broad-line components of $\mathrm{HCN} 4-3$ and $\mathrm{CO} 3-2$, compared with the data. The $\mathrm{CO} / \mathrm{HCN}$ ratio in the broad-line component is therefore $<7$. Note that the upper limit of the $\mathrm{CO} 3-2$ broad-line component contributes $0.3 \mathrm{Jy} \mathrm{km} \mathrm{s}^{-1}$ in the velocity range from -0.2 to $0.4 \mathrm{~km} \mathrm{~s}^{-1}$, which is about $1 / 6$ of the total integrated intensity of $\mathrm{CO} 3-2$ emission.

In order to obtain the $\mathrm{CO} / \mathrm{HCN}$ ratio in the narrow-line component, we first simulate $\mathrm{HCN}$ emission based on the $\mathrm{HCN}$ residual, using the same symmetric outgassing model described above, only with much smaller expansion velocity. However we only fit on the integrated flux based on the spectra, as we cannot reproduce the spatial shift across the nucleus position. Although not ideal, this approach should give a reasonable estimate of the abundance ratio, as long as $\mathrm{CO}$ and $\mathrm{HCN}$ are treated in the same way. Fitting the residual $\mathrm{HCN}$ spectra yields a production rate of $Q_{\mathrm{HCN}}=1.0 \times 10^{26} \mathrm{~s}^{-1}$ and expansion velocity $0.15 \mathrm{~km} \mathrm{~s}^{-1}$. 

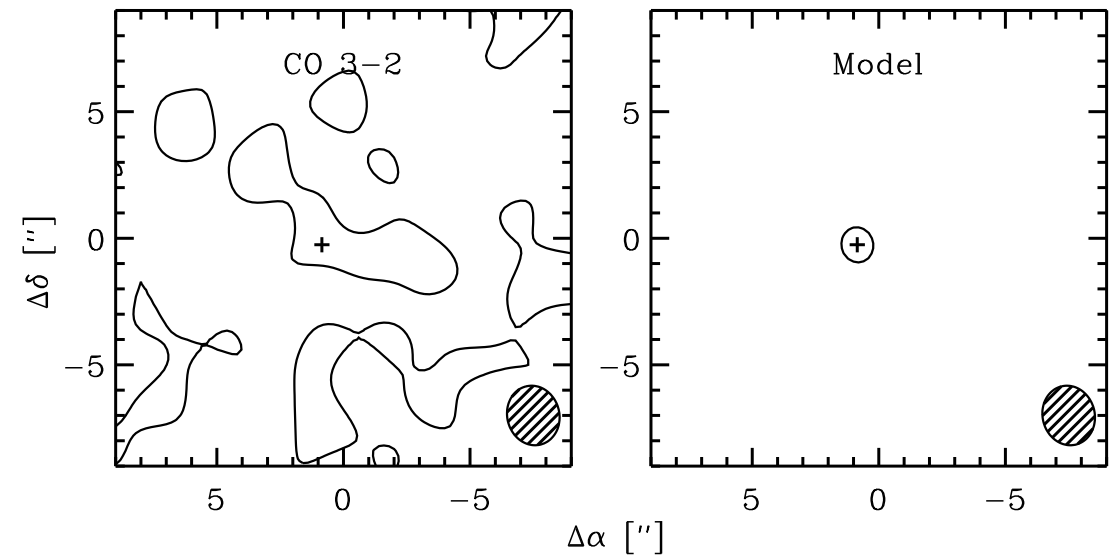

Figure 9. Left: the integrated intensity map of CO 3-2 toward 17P/Holmes on 2007 October 28 UT, integrated between $(-0.8,-0.2)$ and $(0.4,0.8) \mathrm{km} \mathrm{s}^{-1}$; right: the simulated map with $v_{\exp }=0.44 \mathrm{~km} \mathrm{~s}^{-1}$ and $Q_{\mathrm{CO}}=5.0 \times 10^{27} \mathrm{~s}^{-1}$, integrated over the same velocity range. The contour level is $0.08 \mathrm{Jy} \mathrm{beam}^{-1} \mathrm{~km} \mathrm{~s}^{-1}$. The peak of the simulated map matches the rms $\left(0.09 \mathrm{Jy}_{\text {beam }}^{-1} \mathrm{~km} \mathrm{~s}^{-1}\right)$ of the left image, providing the upper limit of the contribution to the CO emission from the broad-line component.

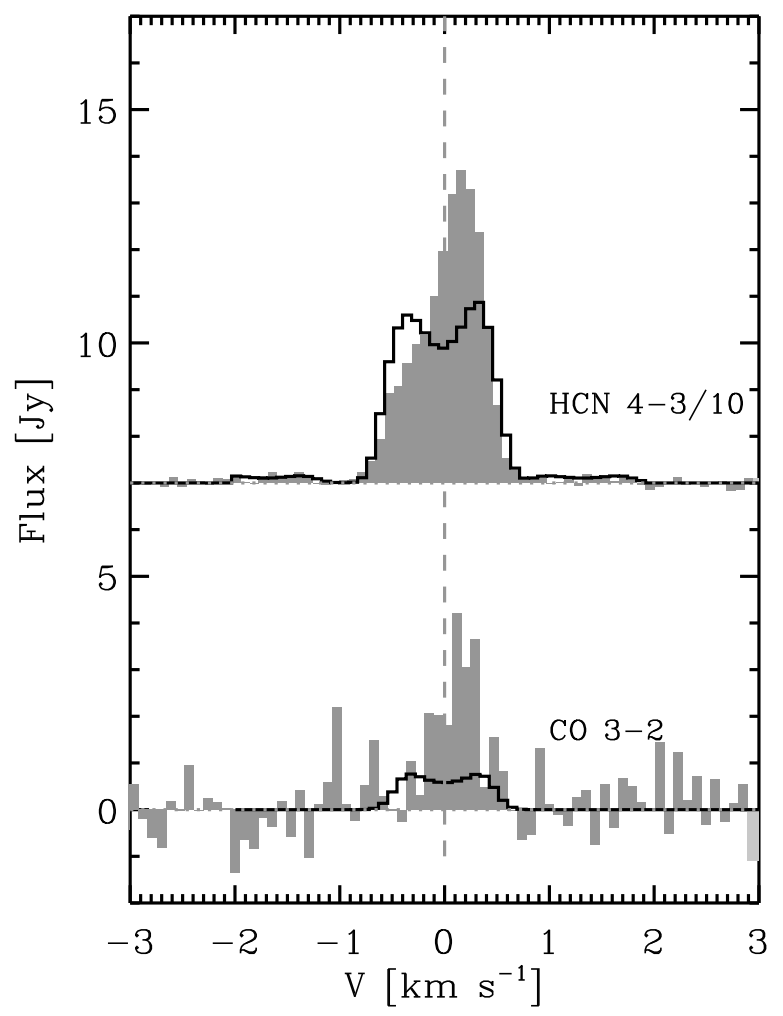

Figure 10. Broad-line components of $\mathrm{HCN} 4-3$ and $\mathrm{CO} 3-2$ emission (upper limit) from 17P/Holmes on 2007 October 28 UT. The solid-line HCN spectra, retrieved from the best-fit symmetric outgassing model with $v_{\exp }=0.44 \mathrm{~km} \mathrm{~s}^{-1}$ and $Q_{\mathrm{HCN}}=8 \times 10^{26} \mathrm{~s}^{-1}$, is overlaid with the data spectra in gray. The solid-line CO spectra is the simulated spectra from the model with $v_{\text {exp }}=0.44 \mathrm{~km} \mathrm{~s}^{-1}$ and $Q_{\mathrm{CO}}=5 \times 10^{27} \mathrm{~s}^{-1}$. The simulated $\mathrm{CO}$ emission model generates the intensity image integrated between $(-0.8,-0.2)$ and $(0.4,0.8) \mathrm{km} \mathrm{s}^{-1}$ as shown in Figure 9, giving the upper limit of the contribution to the $\mathrm{CO}$ emission from the broad-line component. The vertical dashed line marks the cometocentric velocity of $0 \mathrm{~km} \mathrm{~s}^{-1}$.

Assuming there is no contribution of the broad-line component to the CO line, we find $Q_{\mathrm{CO}}=4.5 \times 10^{27} \mathrm{~s}^{-1}$ to match the integrated intensity of $1.75 \mathrm{Jy} \mathrm{km} \mathrm{s}^{-1}$ for CO 3-2 with an expansion velocity of $0.15 \mathrm{~km} \mathrm{~s}^{-1}$. This results in a $\mathrm{CO} /$ $\mathrm{HCN}$ ratio of 45 . If we consider a maximum contribution of the wide component to the $\mathrm{CO}$ line equal to the upper limit derived above, i.e., $0.3 \mathrm{Jy} \mathrm{km} \mathrm{s}^{-1}$, we find $Q_{\mathrm{CO}}=3.5 \times$ $10^{27} \mathrm{~s}^{-1}$ to reproduce the integrated intensity of $1.45 \mathrm{Jy} \mathrm{km} \mathrm{s}^{-1}$,

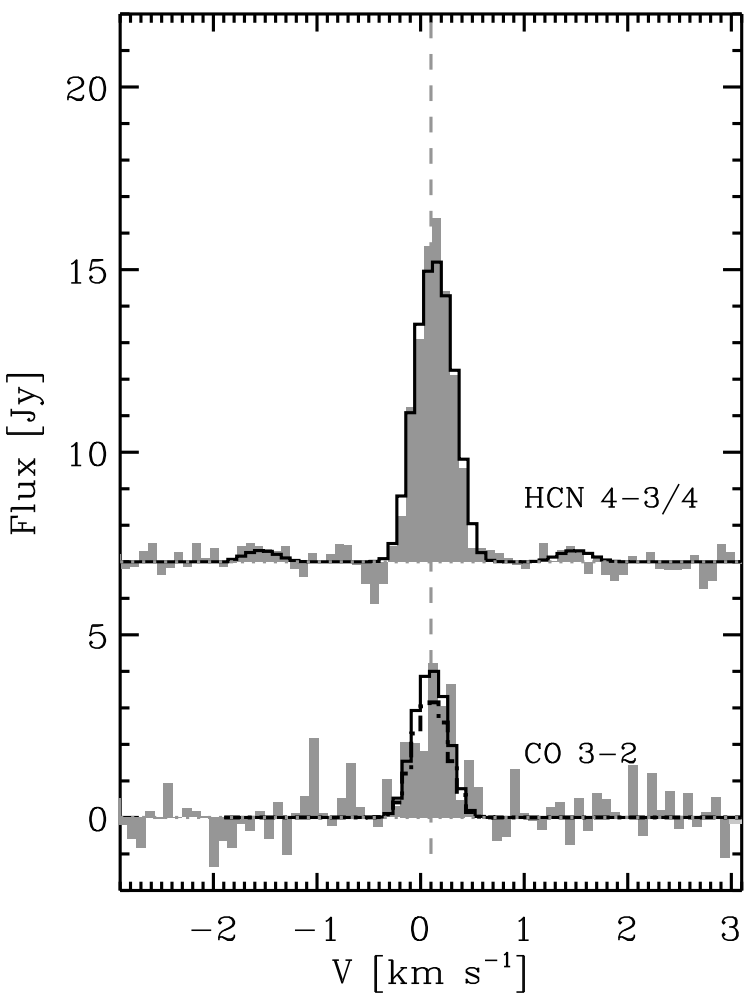

Figure 11. Narrow-line components of $\mathrm{HCN} 4-3$ and $\mathrm{CO} 3-2$ emission from 17P/Holmes on 2007 October 28 UT. The HCN spectra (in gray) is retrieved from the residual of HCN emission subtracted by the best-fit symmetric outgassing model with $v_{\exp }=0.44 \mathrm{~km} \mathrm{~s}^{-1}$ and $Q_{\mathrm{HCN}}=8 \times 10^{26} \mathrm{~s}^{-1}$. The solid-line HCN spectra is the simulated spectra from the model with $v_{\exp }=$ $0.15 \mathrm{~km} \mathrm{~s}^{-1}$ and $Q_{\mathrm{HCN}}=1 \times 10^{26} \mathrm{~s}^{-1}$ which generates the same flux as the spectra in gray. The solid-line $\mathrm{CO}$ spectra are simulated from the model with $v_{\mathrm{exp}}=0.15 \mathrm{~km} \mathrm{~s}^{-1}$ and $Q_{\mathrm{CO}}=4.5 \times 10^{27} \mathrm{~s}^{-1}$ which matches the CO data flux in the gray spectra, $1.75 \mathrm{Jy} \mathrm{km} \mathrm{s}^{-1}$, while the dotted-line CO spectra are simulated with $v_{\exp }=0.15 \mathrm{~km} \mathrm{~s}^{-1}$ and $Q_{\mathrm{CO}}=3.5 \times 10^{27} \mathrm{~s}^{-1}$ which generates the integrated flux $1.35 \mathrm{Jy} \mathrm{km} \mathrm{s}^{-1}$. The vertical dashed line marks the cometocentric velocity of $+0.1 \mathrm{~km} \mathrm{~s}^{-1}$.

and a $\mathrm{CO} / \mathrm{HCN}$ ratio $=35$. Figure 11 shows the narrow-line components of $\mathrm{HCN} 4-3$ and $\mathrm{CO} 3-2$, compared with the $\mathrm{CO}$ data and residual HCN spectra. Since the unknown contribution from the broad-line component dominates the uncertainty of the calculation, we conclude that the ratio of $\mathrm{CO} / \mathrm{HCN}$ in the narrow-line component can be constrained to $40 \pm 5$. This is significantly higher than the $\mathrm{CO} / \mathrm{HCN}<7$ found for the 
broad-line component. Using the same analysis, we determine the ratio $\mathrm{CO} / \mathrm{HCN}<9$ for the broad-line component and $45 \pm 10$ for the narrow-line component on October 26. This is consistent with the result on October 28, despite larger uncertainties due to the fact that the spectral resolution of $\mathrm{HCN}$ and $\mathrm{CO}$ lines carried out on October 26 is a factor of two worse than on October 28, which creates large uncertainties in how much the broad-line component contributes to the $\mathrm{CO}$ emission on October 26.

$\mathrm{HCN}$ is the molecule that exhibits the lowest abundance variation from comet to comet (Biver et al. 2002; Mumma $\&$ Charnley 2011). Therefore, production rates relative to that of $\mathrm{HCN}$ can be used for a comparative study of molecular abundances. From a study of a sample of 24 comets, Biver et al. (2002) found that $\mathrm{CO} / \mathrm{HCN}$ varies from $<24$ to 180. In particular, four long period comets show $\mathrm{CO} / \mathrm{HCN}>85$ while four other comets, including one short period comet, show $\mathrm{CO} / \mathrm{HCN}<40$. However, there is still no obvious correlation between the dynamical class and the chemical composition of comets (Biver et al. 2002; Crovisier, 2007). We find in the near-nucleus outgassing of comet $17 \mathrm{P} /$ Holmes, the $\mathrm{CO} / \mathrm{HCN}$ ratio is much higher in the narrow component than the broad one. The difference of $\mathrm{CO} / \mathrm{HCN}$ ratios in the two components demonstrates the heterogeneity of the nucleus of this Jupiter family comet.

The decrease in the $\mathrm{HCN}$ production rates indicates an $e$ folding time $\sim 4$ days in the October 26 to 29 period (Table 4 ). Schleicher (2009) measured the decline of the production rates of optical species. From their Table 3 we find an $e$-folding time $\sim 14$ days in the 2007 November to 2007 December period, with an indication that the fading timescale increases as time passes up to their last observation in 2008 March. Interestingly, their $\mathrm{CN}$ production rate, $\log _{10}\left(Q_{\mathrm{CN}}\right)=27.4$ on November 1 , is higher than the $\mathrm{HCN}$ production rate $\log _{10}\left(Q_{\mathrm{HCN}}\right)=27.1$ we derived four days earlier (October 28) when matching the whole flux of HCN 4-3 emission. This inequality could be an artifact of the use of the Haser model (which applies to a coma expanding in steady state and which may not accurately describe the outburst coma of 17P) or it could be an indication that $\mathrm{CN}$ has a parent in addition to $\mathrm{HCN}$. Furthermore, the photometer entrance apertures used in the optical measurements, ranging from $24^{\prime \prime}$ to $204^{\prime \prime}$, are different from the primary beams $\left(31^{\prime \prime}\right)$ used in our observations. This could also contribute to the difference in production rates derived for the $\mathrm{CN}$ and $\mathrm{HCN}$ molecules.

\section{DISCUSSION}

What are the two components in the near-nucleus coma of the outbursting 17P/Holmes? As shown from the above model fitting, the HCN emission from the broad-line component can be fit with symmetric outgassing with an expanding velocity about $0.5 \mathrm{~km} \mathrm{~s}^{-1}$. Drahus et al. (2007) and Snodgrass et al. (2007) found that an almost-spherical dust coma expanded around the nucleus of $17 \mathrm{P} /$ Holmes at $0.55 \mathrm{~km} \mathrm{~s}^{-1}$. Both the expansion velocity and symmetric outgassing pattern of the broad-line component appear to be related to this spherical dust coma releasing dust from the surface of the nucleus.

However, within the dust coma, bright streaks emerged from the nucleus, likely produced by fragments that moved away from the nucleus with velocities of up to $0.1 \mathrm{~km} \mathrm{~s}^{-1}$ (Trigo-Rodriguez et al. 2007; Stevenson et al. 2010). Gaillard et al. (2007) also postulated that the streaks originated from disintegrating cometary fragments. The images from Hubble
Telescope's Wide Field Planetary Camera 2 also showed a "bow tie" appearance, ${ }^{5}$ about twice as much dust lies along the east-west direction as along the north-south direction, similar to the morphology we have detected in the narrow-line component. We find the production rate from the broad-line component of HCN 4-3 decreased but the whole integrated intensity of the line actually increased from October 28 to 29, which implies that the narrow-line component should have increased. Coupled with the increase of the mm continuum flux level between the two days, this provides strong evidence for disintegrating cometary fragments continuously supplying the dust and gas near the nucleus. So we believe the narrow-line component detected in the SMA spectral line imaging shown here should be associated with volatiles sublimating from the fragments near the nucleus that was the most recently ejected and most pristine in nature. The main problem with this interpretation is how long these streaks/fragments persisted. Stevenson \& Jewitt (2012) presented the near-nucleus photometry over a period of three months following the outburst of comet $17 \mathrm{P} /$ Holmes and they found a halo consisting of freshly released icy grains persisted around the nucleus and extended to radii of several thousand kilometers, encompassing the emission area we detected. In our interpretation, the narrow-line component likely originated from this halo, with volatiles continuously supplied from the freshly released icy grains. Yang et al. (2009) suggested that only clean ice grains, with $<10 \%$ impurities, could survive long enough to travel $3500 \mathrm{~km}$ from the nucleus before sublimating, which could explain the offsets from the nucleus seen in the narrowline component as in emission of $\mathrm{CO}, \mathrm{CS}$ and $\mathrm{HCN}$ residuals. The line width of the narrow-line component does not change much with time over the four days of observations, compared to the rapid narrowing of the expansion velocity of the symmetric outgassing component. This is consistent with the long lifetime of the halo feature found by Stevenson \& Jewitt (2012).

\section{SUMMARY}

The Jupiter family comet $17 \mathrm{P} /$ Holmes underwent a dramatic outburst in 2007, providing us with a rare opportunity to access fresh material released by the powerful outburst from beneath the thermally processed surface. We have observed 17P/Holmes with the SMA from 2007 October 26 through October 29 at a spatial resolution of $\sim 3000 \mathrm{~km}$ at the comet distance and found peculiar outgassing in the near-nucleus coma. Our key results include the following.

1. We find two components in the molecular emission from the near-nucleus coma: one has relatively broad line width $\left(\sim 1 \mathrm{~km} \mathrm{~s}^{-1}\right.$ FWHM), showing a symmetric outgassing pattern with respect to the nucleus position. The other has a narrow linewidth $\left(<0.5 \mathrm{~km} \mathrm{~s}^{-1}\right.$ FWHM). HCN 4-3 emission has both components, while the emission of CS 7-6 and CO 3-2 is dominated by the narrow-line component.

2. The morphologies of the two components are different: the peaks of the broad-line component in the HCN 4-3 emission are on the nucleus position and do not change with velocity; the line center of the narrow-line component is redshifted by $0.1-0.2 \mathrm{~km} \mathrm{~s}^{-1}$ (cometocentric frame). The peaks of the narrow-line component emission shift with velocity across the nucleus position and the P.A. of the red/ blue gradient changed from $66^{\circ}$ to $30^{\circ}$ during the four days of observations.

\footnotetext{
5 http://www.spacetelescope.org/news/heic0718/
} 
3. We determine distinctly different $\mathrm{CO} / \mathrm{HCN}$ ratios for each of the components: $\mathrm{CO} / \mathrm{HCN}<7$ in the broad-line component and $\mathrm{CO} / \mathrm{HCN}=40 \pm 5$ in the narrow-line one. This different line ratio reflects the heterogeneity of the nucleus of this Jupiter family comet.

4. We determine the ${ }^{12} \mathrm{C} /{ }^{13} \mathrm{C}$ ratio $=80 \pm 16$ based on the simultaneous observations of $\mathrm{HCN}$ and $\mathrm{H}^{13} \mathrm{CN} 4-3$ emission, which is consistent with the terrestrial value.

These observations demonstrate the unique power of radio interferometers with high spatial and spectral resolution to map small scale spatio-kinematic differences in emission of primary volatiles in the near-nucleus cometary comae to probe the heterogeneity in the nucleus. Simultaneous observations of multiple primary volatiles are key to understanding and interpreting the outgassing properties of the nucleus. The newly constructed Atacama Large Millimeter/submillimeter Array (ALMA)/SMA will allow the assessment of the composition of a large sample of comets to examine its relationship with cometary formation region.

The SMA is a joint project between the Smithsonian Astrophysical Observatory and the Academia Sinica Institute of Astronomy and Astrophysics and is funded by the Smithsonian Institution and the Academia Sinica. We thank SMA Director Raymond Blundell for quick allocation of the DDT time for the above observations and the SMA staff, especially Ken (Taco) Young and Nimesh Patel, for observational supports. D.J. acknowledges support from NASA's Origins program.

Facility: SMA

\section{REFERENCES}

Biver, N., Bockelée-Morvan, D., Crovisier, J., et al. 2002, EM\&P, 90,323

Blake, G. A., Qi, C., Hogerheijde, M. R., Gurwell, M. A., \& Muhleman, D. O. 1999, Natur, 398, 213

Bockelée-Morvan, D., Biver, N., Jehin, E., et al. 2008, ApJL, 679, L49

Crovisier, J. 2007, arXiv:astro-ph/0703785

Dello Russo, N., Vervack, R. J., Jr., Weaver, H. A., et al. 2008, ApJ, 680, 793

Ehrenfreund, P., \& Charnley, S. B. 2000, ARA\&A, 38, 427

Gaillard, B., Lecacheux, J., \& Colas, F. 2007, CBET, 1123, 1

Hogerheijde, M. R., Qi, C., de Pater, I., et al. 2009, AJ, 137, 4837

Hogerheijde, M. R., \& van der Tak, F. F. S. 2000, A\&A, 362, 697

Hsieh, H. H., Fitzsimmons, A., Joshi, Y., Christian, D., \& Pollacco, D. L. 2010, MNRAS, 407, 1784

Ishiguro, M., Watanabe, J.-I., Sarugaku, Y., et al. 2010, ApJ, 714, 1324

Jewitt, D., \& Luu, J. 1992, Icar, 100, 187

Jewitt, D., \& Matthews, H. 1999, AJ, 117, 1056

Levison, H. F., \& Duncan, M. J. 1994, Icar, 108, 18

Li, J., Jewitt, D., Clover, J. M., \& Jackson, B. V. 2011, ApJ, 728, 31

Meier, R., Owen, T. C., Jewitt, D. C., et al. 1998, Sci, 279, 1707

Mumma, M. J., \& Charnley, S. B. 2011, ARA\&A, 49, 471

Qi, C. 2001, PhD thesis, California Institute of Technology

Schleicher, D. G. 2009, AJ, 138, 1062

Snodgrass, C., Fitzsimmons, A., Boehnhardt, H., et al. 2007, CBET, 1111,1

Snodgrass, C., Lowry, S. C., \& Fitzsimmons, A. 2006, MNRAS, 373, 1590

Stevenson, R., \& Jewitt, D. 2012, AJ, 144, 138

Stevenson, R., Kleyna, J., \& Jewitt, D. 2010, AJ, 139, 2230

Trigo-Rodriguez, J. M., Abraham, P., Konkoly, A., et al. 2007, CBET, 1118,1

Volk, K., \& Malhotra, R. 2008, ApJ, 687, 714

Yang, B., Jewitt, D., \& Bus, S. J. 2009, AJ, 137, 4538 